\title{
Effect of Annealed Temperature on Microstructure and Magnetic Properties of Ag/FelAg Nanogranular Films
}

\author{
Qin $X U^{1, a, *}$, Ya-Xuan REN ${ }^{1, b}$, Ru-Song $L I^{1, b}$ and Jing-Jin $L I U^{2, c}$ \\ ${ }^{1}$ College of Sciences, Hebei University of Technology, Tianjin 300401, China \\ ${ }^{2}$ Faculty of Science, Tianjin University, Tianjin 300072, China \\ aemail:xuqinzi@126.com, bemail:xq88518408@sina.com, cemail:155291033@qq.com \\ ${ }^{*}$ Corresponding author
}

\section{Keywords: Ag/Fe/Ag, Magnetron sputtering, Coercivity}

\begin{abstract}
Ag}(3 \mathrm{~nm}) / \mathrm{Fe}(35 \mathrm{~nm}) / \operatorname{Ag}(3 \mathrm{~nm})$ nanogranular films were fabricated by magnetron sputtering system onto glass substrates and subsequently annealed at different temperature. The microstructure and magnetic properties of $\mathrm{Ag} / \mathrm{Fe} / \mathrm{Ag}$ films were investigated as the annealed temperature increases from room temperature to $500{ }^{\circ} \mathrm{C}$. The XRD patterns indicate that annealing disposal does not change crystal structure of the films. The SPM was used to get the morphology and magnetic domain structures, the SPM results reveal that the annealed temperature has a great effect on the average grain size. The VSM results show that out-of-plane coercivity reaches maximum about $3.3 \mathrm{kOe}$ after annealed at $500{ }^{\circ} \mathrm{C}$. The result of $\delta \mathrm{M}$ plot indicates that the intergranular interactions are mainly of magnetostatic interaction. It concludes that the reduction of exchange interaction and the enhancement of coercivity, which maybe result from the nonmagnetic silver atoms, diffuse into the grain boundaries of magnetic grains during annealing.
\end{abstract}

\section{Introduction}

Nanosized magnets with various shape and size have long attracted the attention of scientific researchers due to their potential for practical application. In recent years, a large amount of experimental work, especially on the investigation of Fe-base and Co-base alloy material[1-5], has been undertaken to search new materials which have high coercivity, high magnetic anisotropy, good corrosion resistance and large energy products.

Recently, considerable attention has been paid to the investigation of Fe-base films due to their cost reduction. According to relevant literature reports, nonmagnetic particle coating in magnetic particle boundary can effectively reduce the noise of medium, and then improve its microstructure and magnetic properties. It has been reported that $\mathrm{Ag}$ is a good buffer layer for FePt and CoPt recording media [6,7]. Peng et al. reported the effects of Ag layer thickness on magnetic properties and microstructure of $\mathrm{Ag} / \mathrm{Fe} / \mathrm{Ag}$ granular films [8]. It is speculated that $\mathrm{Ag}$ is probably also a kind of ideal buffer layer material for Fe medium. Therefore, it is reasonable that the nonmagnetic Ag atoms might diffuse into the grain boundaries of magnetic Fe grains during annealing process, and thereby leading to highly coercivity, highly magnetic anisotropy.

It is worth noting that the annealed temperature also has an important effect on microstructure and magnetic properties of $\mathrm{Ag} / \mathrm{Fe} / \mathrm{Ag}$ nanogranular films. In the present paper, we report the fabrication of $\mathrm{Ag}(3 \mathrm{~nm}) / \mathrm{Fe}(35 \mathrm{~nm}) / \mathrm{Ag}(3 \mathrm{~nm})$ films by magnetron sputtering system, and also discuss the effect of annealed temperature on microstructure and magnetic properties of such films using SPM、XRD and VSM.

\section{Experimental Works}

$\mathrm{Ag}(3 \mathrm{~nm}) / \mathrm{Fe}(35 \mathrm{~nm}) / \mathrm{Ag}(3 \mathrm{~nm})$ films were deposited onto glass substrates using a DC facing-target magnetron sputtering system. The base pressure was $1.5 \times 10^{-5} \mathrm{~Pa}$ and argon working pressure was 0.5 
Pa. Subsequently, the obtained samples were in situ annealed at a temperature ranging from $200{ }^{\circ} \mathrm{C}$ to $500{ }^{\circ} \mathrm{C}$ for $30 \mathrm{~min}$ in vacuum.

The magnetic properties of the films were measured with vibrating sample magnetometer (VSM) at room temperature. The atomic force microscopy (AFM) and magnetic force microscopy (MFM) images were scanned using scanning probe microscope (SPM). The crystal structure of the films was analyzed by $\mathrm{x}$-ray diffraction (XRD) with $\mathrm{Cu} \mathrm{Ka}$ radiation. $\delta \mathrm{M}$ plot was used to give a direct survey of intergranular interaction between neighboring grains[9].

\section{Results and Discussion}

(1) The analysis of the crystal structure of the films
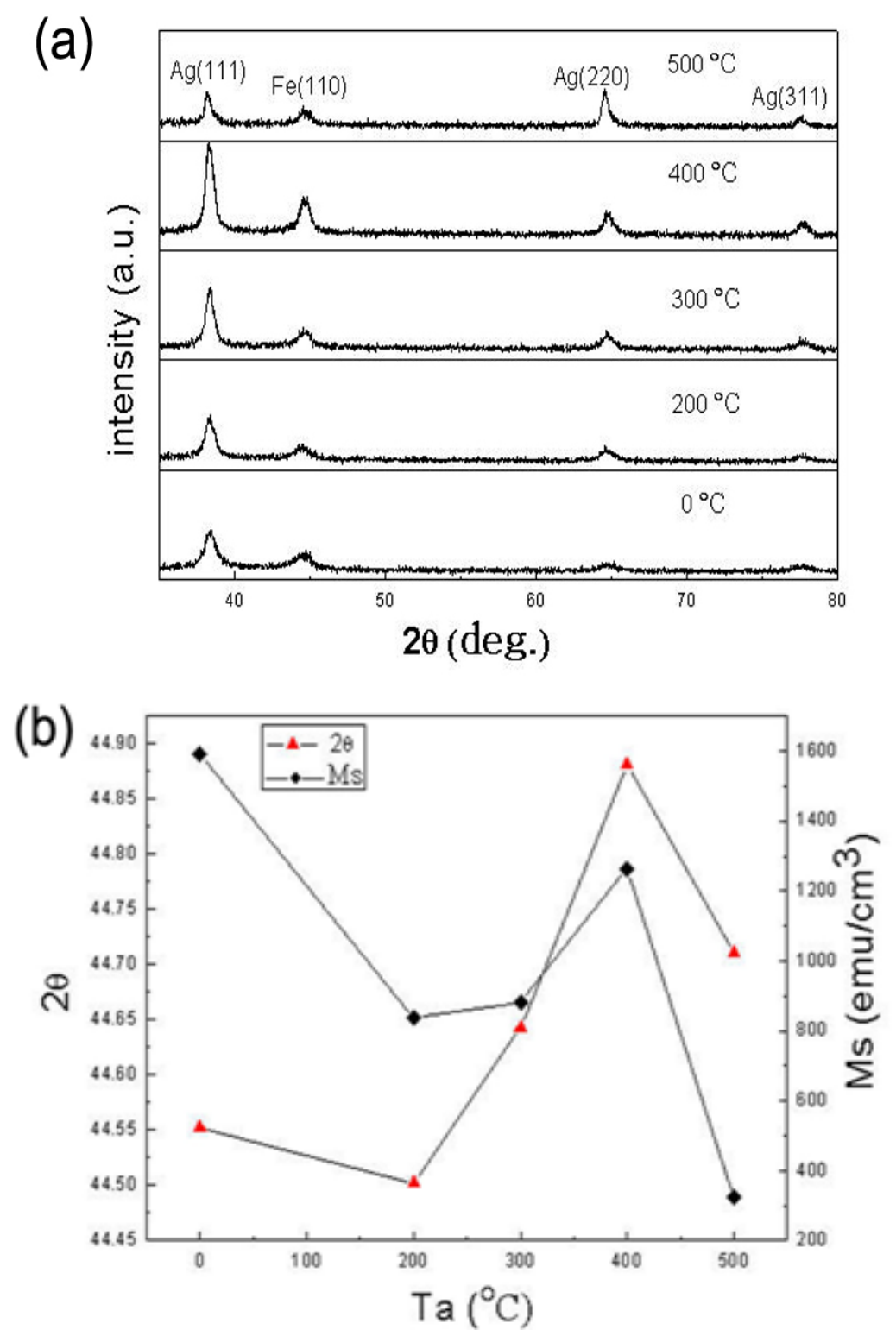

Fig.1. XRD patterns (a) and the corresponding $2 \theta$ values of $\mathrm{Fe}(110)$ and Ms (b) of the films with different $\mathrm{T}_{\mathrm{a}}$

Fig.1a shows the XRD patterns of $\mathrm{Ag}(3 \mathrm{~nm}) / \mathrm{Fe}(35 \mathrm{~nm}) / \mathrm{Ag}(3 \mathrm{~nm})$ films annealed at various temperatures. The patterns show that all the films can observe FCC Ag(111) peak and BCC Fe(110) peak, which clearly indicates that annealing disposal does not change crystal structure of the films. As annealed temperature is increased, the $2 \theta$ values of $\mathrm{Fe}(110)$ peak have a slightly shift, the change trend shown in Figure 1b. It indicates that annealing disposal can change the lattice constant of the film. It is known that the lattice constant is increased as the $2 \theta$ values of $\mathrm{Fe}(110)$ peak decreased. We 
conclude that as-deposited film has not interpenetration between Ag and Fe layer grain, so lattice constant quite small. After annealed at $200{ }^{\circ} \mathrm{C}$, tiny thermal disturbance makes interparticle spacing increase, leading to increase of lattice constant. As annealed temperature is increased to $300{ }^{\circ} \mathrm{C}$ or $400{ }^{\circ} \mathrm{C}$, the nonmagnetic Ag atoms diffuse into the magnetic Fe grain boundary to reduce lattice constant. However, annealed at $500{ }^{\circ} \mathrm{C}$, Ag atoms are separated from Fe layer as its low surface energy, results to the increase of lattice constant again. It is worth mentioning that the increase of lattice constant can arose the decrease of saturation magnetization(Ms), and this indicates that $2 \theta$ values of $\mathrm{Fe}(110)$ and Ms should have a same change trend as shown in Fig1b.

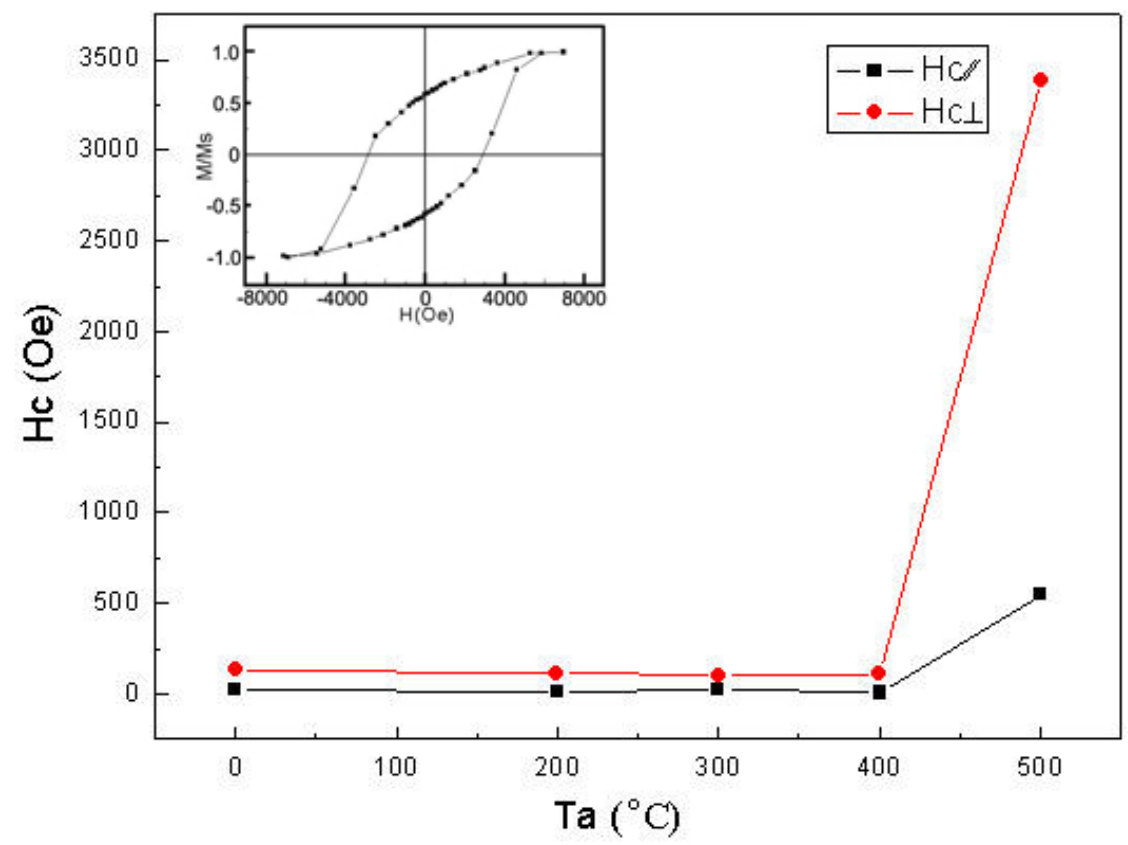

Fig.2. Coercivity of the film with different annealed temperature $T_{a}$, the inset is out-of-plane hysteresis loops of annealed at $500^{\circ} \mathrm{C}$ of the film.

(2) The analysis of the magnetic properties of the films

Fig. 2 shows in-plane and out-of-plane coercivity of $\mathrm{Ag}(3 \mathrm{~nm}) / \mathrm{Fe}(35 \mathrm{~nm}) / \mathrm{Ag}(3 \mathrm{~nm})$ films change as a function of annealed temperature. We can see that, the change of in-plane and out-of-plane coercivity first smoothly changes then sharply increases with increasing annealed temperature. The coercivity of in-plane and out-of-plane both reaches maximum after annealed at $500^{\circ} \mathrm{C}$, out-of-plane coercivity reaches to $3.3 \mathrm{kOe}$. The inset is out-of-plane hysteresis loops of annealed at $500^{\circ} \mathrm{C}$ of the film. The reason for obtained such highly coercivity may be attributed to that Ag under-layers separate island-like grain distributing on substrates and Ag cap-layers move into Fe layers properly at right temperature, which effectively reduce the exchange-coupling interaction between grains. It is confirmed by $\delta \mathrm{M}(\mathrm{H})$ plot[10] as shown in Fig. 3. 


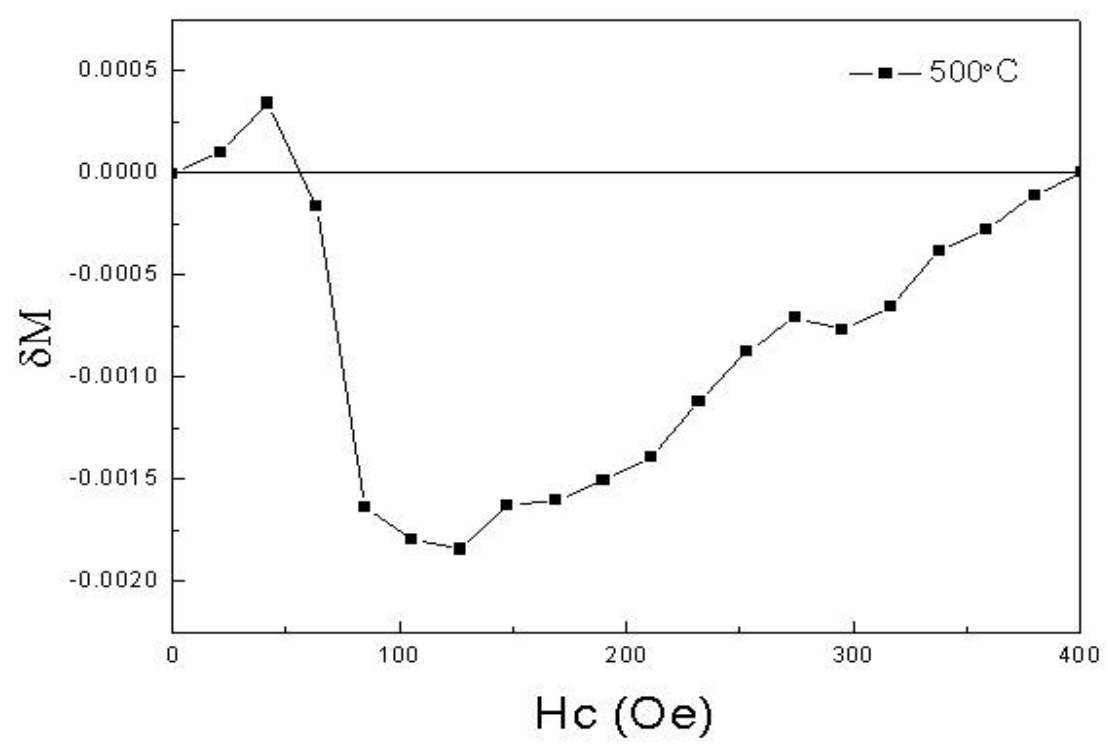

Fig.3. $\delta \mathrm{M}$ plot of the film annealed at $500^{\circ} \mathrm{C}$

According to correlative literatures report, magnetic intergranular interactions have a great effect on macro-magnetism of materials. Intergranular interactions can describe by $\delta \mathrm{M}(\mathrm{H})$ plot. So we measure dc demagnetization remanence measurement (DCD) and isothermal remanence magnetization (IRM) using VSM, and calculate $\delta \mathrm{M}(\mathrm{H})$ plot shown in Fig.3. $\delta \mathrm{M}(\mathrm{H})$ plot of the film annealed at $500{ }^{\circ} \mathrm{C}$ has positive and negative peak. But negative peak is larger, it implies that intergranular interactions is mainly of magnetostatic interaction. It maybe attributes to the nonmagnetic silver atoms diffuse into the grain boundaries of magnetic grains during annealing, which is also the reason for highly coercivity of the film annealed at $500{ }^{\circ} \mathrm{C}$.
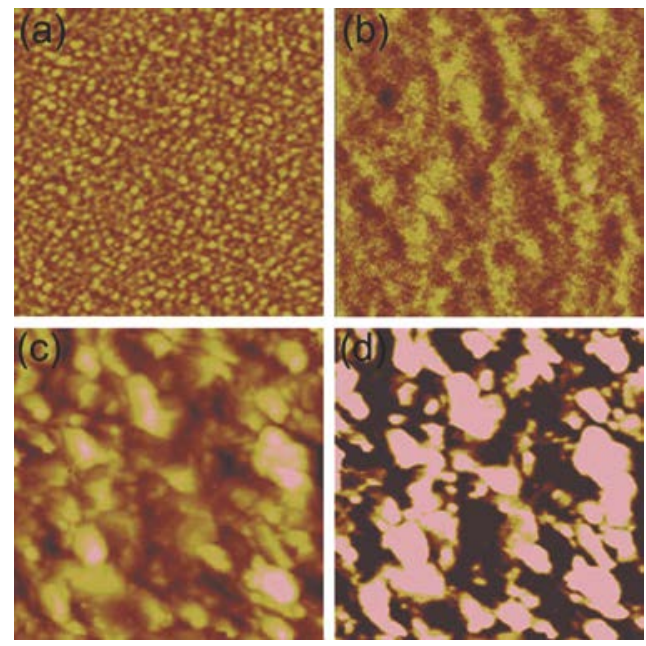

Fig.4. AFM (left) and MFM (right) images of the film, scan size is $1 \mu \mathrm{m} \times 1 \mu \mathrm{m}$,

(a) and (b) as-deposited, (c) and (d) $\mathrm{T}_{\mathrm{a}}=500{ }^{\circ} \mathrm{C}$

(3) The analysis of the morphology of the films

Further characterization of the above results was carried out by SPM. For MFM images, dark and light regions represent the magnetic clusters in which the magnetic moments align in the same direction, parallel or perpendicular to the film normal. Figure 4 shows AFM and MFM images of the films with as-deposited and annealed at $500{ }^{\circ} \mathrm{C}$. MFM images scanned the same regions with AFM images of the $\mathrm{Ag}(3 \mathrm{~nm}) / \mathrm{Fe}(35 \mathrm{~nm}) / \mathrm{Ag}(3 \mathrm{~nm})$ thin films. The as-deposited film has a small grain with uniform distribution. Annealed at $500{ }^{\circ} \mathrm{C}$, the average grain size and the maximum average roughness 
are increased to $14 \mathrm{~nm}$ and $1.2 \mathrm{~nm}$, while the average magnetic domain size is decreased. The above results reveal that the annealed temperature has a great effect on the average grain size and the maximum average roughness. In addition, roughness has a great effect on coercivity, usually coercivity is higher when roughness is larger. In our experiment, roughness reaches maximum after annealed at $500{ }^{\circ} \mathrm{C}$, the corresponding coercivity also reaches to maximum, which is in good agreement with magnetic measure.

\section{Conclusions}

$\mathrm{Ag}(3 \mathrm{~nm}) / \mathrm{Fe}(35 \mathrm{~nm}) / \mathrm{Ag}(3 \mathrm{~nm})$ films were prepared by magnetron sputtering, and subsequently annealed at various temperature. The XRD patterns show that all the films observed FCC Ag(111) peak and BCC Fe(110) peak. The as-deposited film has a small grain with uniform distribution, the roughness of the films reaches maximum after annealed at $500{ }^{\circ} \mathrm{C}$. The coercivity of the films increases from 200 Oe to $3.3 \mathrm{KOe}$ as the annealing temperature increases from room temperature to $500{ }^{\circ} \mathrm{C}$. $\delta \mathrm{M}$ plot indicates that intergranular interaction is mainly of magnetostatic interaction. It maybe attributes to the nonmagnetic silver atoms diffuse into the grain boundaries of magnetic grains during annealing.

\section{Acknowledgments}

This work is supported by the National Natural Sciences Foundation of China (Grant No. 11547250), the Natural Science Foundation of Hebei Province (Grant No. A2015202343), and the Research Project of Hebei Science and Technology Department (Grant No. 15211043). The authors wish to thank Dr. Norm Davison for helpful discussion.

\section{References}

[1] X.X. Liu, A. Morisako, Soft magnetic properties of FeCo films with high saturation magnetization, J. Appl. Phys. 103 (2008) 07E726.

[2] G. Chen, J. Zhu, A. Quesada, J. Li, A.T. N'diaye, Y. Huo, T.P. Ma, Y. Chen, H.Y. Kwon, C. Won, Z.Q. Qiu, A.K. Schmid, Y.Z. Wu, Novel chiral magnetic domain wall structure in Fe/Ni/Cu(001) films, Phys. Rev. Lett. 110 (2013) 177204.

[3] D.S. Hung, C.S. Tsai, C.C. Yu, Y. Liou, S.F. Lee, P.C. Chiang, C.S. Ho, Y.D. Yao, Microwave switching behaviors of Fe/Ag/Fe/Ag epitaxial films, J. Magn. Magn. Mater. 304 (2006) e118-e120.

[4] H. Kockar, M. Alper, T. Sahin, O. Karaagac, Role of electrolyte pH on structural and magnetic properties of Co-Fe films, J. Magn. Magn. Mater. 322 (2010) 1095-1097.

[5] D. Goll, A. Breitling, Coercivity of ledge-type $\mathrm{L} 1_{0}-\mathrm{FePt} / \mathrm{Fe}$ nanocomposites with perpendicular magnetization, Appl. Phys. Lett. 94 (2009) 052502.

[6] P. Allia, F. Celegato, M. Coïsson, P. Tiberto, F. Vinai, F. Albertini, F. Casoli, S. Fabbrici, Effect of $\mathrm{Ag}$ addition on the magnetic and magnetoresistance properties of $\mathrm{Fe}_{53} \mathrm{Pt}_{47}$ films, J. Magn. Magn. Mater. 316 (2007) e35-e39.

[7] C. Feng, B. H. Li, G. Han, J. Teng, Y. Jiang, T. Yang, G. H. Yu, Effect of the underlayer (Ag, Ti or $\mathrm{Bi}$ ) on the magnetic properties of Fe/Pt multilayer films, Thin Solid Films, 515 (2007) 8009-8012.

[8] Y. Peng, Y. Y. Hu, Y. P. Li, H. Y. Sun, Microstructure and magnetic properties of Ag/Fe/Ag pseudo-sandwich nanogranular films, Chin. J. Chem. Phys. 20 (2008) 773-778.

[9] E. P. Wohlfarth, Relations between different modes of acquisition of the remanent magnetization of ferromagnetic particles, J. Appl. Phys. 29 (1958) 595. 
[10] S. K. Chen, F. T. Yuan, W. C. Chang, T. S. Chin, Magnetic properties of FePt and $\mathrm{Fe}_{50} \mathrm{Pt}_{50-x} \mathrm{Nb}_{x}(x=0.00,0.83,1.31,2.05)$ thin films, J. Magn. Magn. Mater. 239 (2002) 471-474. 